\title{
Several Classes of BCI-algebras and their Properties
}

\author{
Yuzhong Ding \\ Qingdao University of Science \\ and Technology \\ China
}

Summary. I have formalized the BCI-algebras closely following the book [6], sections 1.1 to $1.3,1.6,2.1$ to 2.3 , and 2.7. In this article the general theory of BCI-algebras and several classes of BCI-algebras are given.

MML identifier: BCIALG_1, version: 7.8.04 4.81.962

The articles [10], [4], [13], [9], [3], [12], [2], [11], [5], [7], [8], [1], and [14] provide the notation and terminology for this paper.

\section{The Basics of General Theory of BCI-algebras}

We introduce BCI structures which are extensions of 1-sorted structure and are systems

$\langle$ a carrier, an internal complement $\rangle$,

where the carrier is a set and the internal complement is a binary operation on the carrier.

Let us note that there exists a BCI structure which is non empty and strict.

Let $A$ be a BCI structure and let $x, y$ be elements of $A$. The functor $x \backslash y$ yielding an element of $A$ is defined by:

(Def. 1) $x \backslash y=$ (the internal complement of $A)(x, y)$.

We introduce BCI structures with 0 which are extensions of BCI structure and zero structure and are systems

$\langle$ a carrier, an internal complement, a zero $\rangle$, 
where the carrier is a set, the internal complement is a binary operation on the carrier, and the zero is an element of the carrier.

Let us note that there exists a BCI structure with 0 which is non empty and strict.

Let $I_{1}$ be a non empty BCI structure with 0 and let $x$ be an element of $I_{1}$. The functor $x^{\mathrm{c}}$ yields an element of $I_{1}$ and is defined by:

(Def. 2) $x^{\mathrm{c}}=0_{\left(I_{1}\right)} \backslash x$.

Let $I_{1}$ be a non empty BCI structure with 0 . We say that $I_{1}$ is B if and only if:

(Def. 3) For all elements $x, y, z$ of $I_{1}$ holds $x \backslash y \backslash(z \backslash y) \backslash(x \backslash z)=0_{\left(I_{1}\right)}$.

We say that $I_{1}$ is $\mathrm{C}$ if and only if:

(Def. 4) For all elements $x, y, z$ of $I_{1}$ holds $x \backslash y \backslash z \backslash(x \backslash z \backslash y)=0_{\left(I_{1}\right)}$.

We say that $I_{1}$ is I if and only if:

(Def. 5) For every element $x$ of $I_{1}$ holds $x \backslash x=0_{\left(I_{1}\right)}$.

We say that $I_{1}$ is $\mathrm{K}$ if and only if:

(Def. 6) For all elements $x, y$ of $I_{1}$ holds $x \backslash y \backslash x=0_{\left(I_{1}\right)}$.

We say that $I_{1}$ is BCI- 4 if and only if:

(Def. 7) For all elements $x, y$ of $I_{1}$ such that $x \backslash y=0_{\left(I_{1}\right)}$ and $y \backslash x=0_{\left(I_{1}\right)}$ holds $x=y$.

We say that $I_{1}$ is BCK-5 if and only if:

(Def. 8) For every element $x$ of $I_{1}$ holds $x^{\mathrm{c}}=0_{\left(I_{1}\right)}$.

The BCI structure BCI-EXAMPLE with 0 is defined as follows:

(Def. 9) BCI-EXAMPLE $=\left\langle\{\emptyset\}, \mathrm{op}_{2}, \mathrm{op}_{0}\right\rangle$.

Let us note that BCI-EXAMPLE is strict and non empty.

One can verify that there exists a non empty BCI structure with 0 which is strict, B, C, I, and BCI-4.

A BCI-algebra is B C I BCI-4 non empty BCI structure with 0 .

Let $X$ be a BCI-algebra. A BCI-algebra is called a subalgebra of $X$ if it satisfies the conditions (Def. 10).

(Def. 10)(i) $\quad 0_{\mathrm{it}}=0_{X}$,

(ii) the carrier of it $\subseteq$ the carrier of $X$, and

(iii) the internal complement of it $=$ (the internal complement of $X) \uparrow$ (the carrier of it).

The following proposition is true

(1) Let $X$ be a non empty BCI structure with 0 . Then $X$ is a BCI-algebra if and only if the following conditions are satisfied:

(i) $X$ is I and BCI-4, and

(ii) for all elements $x, y, z$ of $X$ holds $x \backslash y \backslash(x \backslash z) \backslash(z \backslash y)=0_{X}$ and $x \backslash(x \backslash y) \backslash y=0_{X}$. 
One can check that there exists a BCI-algebra which is strict and BCK-5.

A BCK-algebra is BCK-5 BCI-algebra.

Let $I_{1}$ be a non empty BCI structure with 0 and let $x, y$ be elements of $I_{1}$. The predicate $x \leq y$ is defined as follows:

(Def. 11) $x \backslash y=0_{\left(I_{1}\right)}$.

We use the following convention: $X$ denotes a BCI-algebra, $x, y, z, u, a, b$ denote elements of $X$, and $I_{1}$ denotes a non empty subset of $X$.

We now state a number of propositions:

(2) $x \backslash 0_{X}=x$.

(3) If $x \backslash y=0_{X}$ and $y \backslash z=0_{X}$, then $x \backslash z=0_{X}$.

(4) If $x \backslash y=0_{X}$, then $x \backslash z \backslash(y \backslash z)=0_{X}$ and $z \backslash y \backslash(z \backslash x)=0_{X}$.

(5) If $x \leq y$, then $x \backslash z \leq y \backslash z$ and $z \backslash y \leq z \backslash x$.

(6) If $x \backslash y=0_{X}$, then $(y \backslash x)^{\mathrm{c}}=0_{X}$.

(7) $x \backslash y \backslash z=x \backslash z \backslash y$.

(8) $x \backslash(x \backslash(x \backslash y))=x \backslash y$.

(9) $\quad(x \backslash y)^{\mathrm{c}}=x^{\mathrm{c}} \backslash y^{\mathrm{c}}$.

(10) $x \backslash(x \backslash y) \backslash(y \backslash x) \backslash(x \backslash(x \backslash(y \backslash(y \backslash x))))=0_{X}$.

(11) Let $X$ be a non empty BCI structure with 0 . Then $X$ is a BCI-algebra if and only if the following conditions are satisfied:

(i) $X$ is BCI-4, and

(ii) for all elements $x, y, z$ of $X$ holds $x \backslash y \backslash(x \backslash z) \backslash(z \backslash y)=0_{X}$ and $x \backslash 0_{X}=x$.

(12) If for every BCI-algebra $X$ and for all elements $x, y$ of $X$ holds $x \backslash(x \backslash y)=$ $y \backslash(y \backslash x)$, then $X$ is a BCK-algebra.

(13) If for every BCI-algebra $X$ and for all elements $x, y$ of $X$ holds $x \backslash y \backslash y=$ $x \backslash y$, then $X$ is a BCK-algebra.

(14) If for every BCI-algebra $X$ and for all elements $x, y$ of $X$ holds $x \backslash(y \backslash x)=$ $x$, then $X$ is a BCK-algebra.

(15) If for every BCI-algebra $X$ and for all elements $x, y, z$ of $X$ holds $(x \backslash$ $y) \backslash y=x \backslash z \backslash(y \backslash z)$, then $X$ is a BCK-algebra.

(16) If for every BCI-algebra $X$ and for all elements $x, y$ of $X$ holds $x \backslash y \backslash$ $(y \backslash x)=x \backslash y$, then $X$ is a BCK-algebra.

(17) If for every BCI-algebra $X$ and for all elements $x, y$ of $X$ holds $x \backslash y \backslash$ $(x \backslash y \backslash(y \backslash x))=0_{X}$, then $X$ is a BCK-algebra.

(18) For every BCI-algebra $X$ holds $X$ is $\mathrm{K}$ iff $X$ is a BCK-algebra.

Let $X$ be a BCI-algebra. The functor BCK-part $X$ yielding a non empty subset of $X$ is defined by:

(Def. 12) BCK-part $X=\left\{x ; x\right.$ ranges over elements of $\left.X: 0_{X} \leq x\right\}$. 
Next we state the proposition

(19) $0_{X} \in$ BCK-part $X$.

Let us consider $X$. Note that $0_{X}$

Next we state three propositions:

(20) For all elements $x, y$ of BCK-part $X$ holds $x \backslash y \in$ BCK-part $X$.

(21) For every element $x$ of $X$ and for every element $y$ of BCK-part $X$ holds $x \backslash y \leq x$.

(22) $X$ is a subalgebra of $X$.

Let $X$ be a BCI-algebra and let $I_{1}$ be a subalgebra of $X$. We say that $I_{1}$ is proper if and only if:

(Def. 13) $\quad I_{1} \neq X$.

Let us consider $X$. Note that there exists a subalgebra of $X$ which is non proper.

Let $X$ be a BCI-algebra and let $I_{1}$ be an element of $X$. We say that $I_{1}$ is atom if and only if:

(Def. 14) For every element $z$ of $X$ such that $z \backslash I_{1}=0_{X}$ holds $z=I_{1}$.

Let $X$ be a BCI-algebra. The functor AtomSet $X$ yields a non empty subset of $X$ and is defined by:

(Def. 15) AtomSet $X=\{x ; x$ ranges over elements of $X: x$ is atom $\}$.

One can prove the following propositions:

(23) $0_{X} \in$ AtomSet $X$.

(24) For every element $x$ of $X$ holds $x \in$ AtomSet $X$ iff for every element $z$ of $X$ holds $z \backslash(z \backslash x)=x$.

(25) For every element $x$ of $X$ holds $x \in$ AtomSet $X$ iff for all elements $u, z$ of $X$ holds $z \backslash u \backslash(z \backslash x)=x \backslash u$.

(26) For every element $x$ of $X$ holds $x \in$ AtomSet $X$ iff for all elements $y, z$ of $X$ holds $x \backslash(z \backslash y) \leq y \backslash(z \backslash x)$.

(27) For every element $x$ of $X$ holds $x \in$ AtomSet $X$ iff for all elements $y, z$, $u$ of $X$ holds $(x \backslash u) \backslash(z \backslash y) \leq y \backslash u \backslash(z \backslash x)$.

(28) For every element $x$ of $X$ holds $x \in$ AtomSet $X$ iff for every element $z$ of $X$ holds $z^{\mathrm{c}} \backslash x^{\mathrm{c}}=x \backslash z$.

(29) For every element $x$ of $X$ holds $x \in$ AtomSet $X$ iff $\left(x^{\mathrm{c}}\right)^{\mathrm{c}}=x$.

(30) For every element $x$ of $X$ holds $x \in$ AtomSet $X$ iff for every element $z$ of $X$ holds $(z \backslash x)^{\mathrm{c}}=x \backslash z$.

(31) For every element $x$ of $X$ holds $x \in$ AtomSet $X$ iff for every element $z$ of $X$ holds $\left((x \backslash z)^{\mathrm{c}}\right)^{\mathrm{c}}=x \backslash z$.

(32) For every element $x$ of $X$ holds $x \in$ AtomSet $X$ iff for all elements $z, u$ of $X$ holds $z \backslash(z \backslash(x \backslash u))=x \backslash u$. 
(33) For every element $a$ of AtomSet $X$ and for every element $x$ of $X$ holds $a \backslash x \in$ AtomSet $X$.

Let $X$ be a BCI-algebra and let $a, b$ be elements of AtomSet $X$. Then $a \backslash b$ is an element of AtomSet $X$.

One can prove the following propositions:

(34) For every element $x$ of $X$ holds $x^{\text {c }} \in$ AtomSet $X$.

(35) For every element $x$ of $X$ there exists an element $a$ of AtomSet $X$ such that $a \leq x$.

Let $X$ be a BCI-algebra. We say that $X$ is generated by atom if and only if:

(Def. 16) For every element $x$ of $X$ there exists an element $a$ of AtomSet $X$ such that $a \leq x$.

Let $X$ be a BCI-algebra and let $a$ be an element of AtomSet $X$. The functor BranchV $a$ yields a non empty subset of $X$ and is defined as follows:

(Def. 17) BranchV $a=\{x ; x$ ranges over elements of $X: a \leq x\}$.

We now state several propositions:

(36) Every BCI-algebra is generated by atom.

(37) For all elements $a, b$ of AtomSet $X$ and for every element $x$ of BranchV $b$ holds $a \backslash x=a \backslash b$.

(38) For every element $a$ of AtomSet $X$ and for every element $x$ of BCK-part $X$ holds $a \backslash x=a$.

(39) For all elements $a, b$ of AtomSet $X$ and for every element $x$ of BranchV $a$ and for every element $y$ of BranchV $b$ holds $x \backslash y \in \operatorname{BranchV}(a \backslash b)$.

(40) For every element $a$ of AtomSet $X$ and for all elements $x, y$ of BranchV $a$ holds $x \backslash y \in$ BCK-part $X$.

(41) For all elements $a, b$ of AtomSet $X$ and for every element $x$ of BranchV $a$ and for every element $y$ of BranchV $b$ such that $a \neq b$ holds $x \backslash y \notin$ BCK-part $X$.

(42) For all elements $a, b$ of AtomSet $X$ such that $a \neq b$ holds BranchV $a \cap$ BranchV $b=\emptyset$.

Let $X$ be a BCI-algebra. A non empty subset of $X$ is said to be an ideal of $X$ if:

(Def. 18) $0_{X} \in$ it and for all elements $x, y$ of $X$ such that $x \backslash y \in$ it and $y \in$ it holds $x \in$ it.

Let $X$ be a BCI-algebra and let $I_{1}$ be an ideal of $X$. We say that $I_{1}$ is closed if and only if:

(Def. 19) For every element $x$ of $I_{1}$ holds $x^{\mathrm{c}} \in I_{1}$.

Let us consider $X$. Note that there exists an ideal of $X$ which is closed.

Next we state four propositions:

(43) $\left\{0_{X}\right\}$ is a closed ideal of $X$. 
(44) The carrier of $X$ is a closed ideal of $X$.

(45) BCK-part $X$ is a closed ideal of $X$.

(46) If $I_{1}$ is an ideal of $X$, then for all elements $x, y$ of $X$ such that $x \in I_{1}$ and $y \leq x$ holds $y \in I_{1}$.

\section{Associative BCI-Algebras}

Let $I_{1}$ be a BCI-algebra. We say that $I_{1}$ is associative if and only if:

(Def. 20) For all elements $x, y, z$ of $I_{1}$ holds $(x \backslash y) \backslash z=x \backslash(y \backslash z)$.

We say that $I_{1}$ is quasi-associative if and only if:

(Def. 21) For every element $x$ of $I_{1}$ holds $\left(x^{\mathrm{c}}\right)^{\mathrm{c}}=x^{\mathrm{c}}$.

We say that $I_{1}$ is positive-implicative if and only if:

(Def. 22) For all elements $x, y$ of $I_{1}$ holds $(x \backslash(x \backslash y)) \backslash(y \backslash x)=x \backslash(x \backslash(y \backslash(y \backslash x)))$.

We say that $I_{1}$ is weakly-positive-implicative if and only if:

(Def. 23) For all elements $x, y, z$ of $I_{1}$ holds $(x \backslash y) \backslash z=x \backslash z \backslash z \backslash(y \backslash z)$.

We say that $I_{1}$ is implicative if and only if:

(Def. 24) For all elements $x, y$ of $I_{1}$ holds $(x \backslash(x \backslash y)) \backslash(y \backslash x)=y \backslash(y \backslash x)$.

We say that $I_{1}$ is weakly-implicative if and only if:

(Def. 25) For all elements $x, y$ of $I_{1}$ holds $x \backslash(y \backslash x) \backslash(y \backslash x)^{\mathrm{c}}=x$.

We say that $I_{1}$ is $p$-semisimple if and only if:

(Def. 26) For all elements $x, y$ of $I_{1}$ holds $x \backslash(x \backslash y)=y$.

We say that $I_{1}$ is alternative if and only if:

(Def. 27) For all elements $x, y$ of $I_{1}$ holds $x \backslash(x \backslash y)=(x \backslash x) \backslash y$ and $(x \backslash y) \backslash y=$ $x \backslash(y \backslash y)$.

One can check that there exists a BCI-algebra which is implicative, positiveimplicative, $p$-semisimple, associative, weakly-implicative, and weakly-positiveimplicative.

Next we state several propositions:

(47) $X$ is associative iff for every element $x$ of $X$ holds $x^{\mathrm{c}}=x$.

(48) For all elements $x, y$ of $X$ holds $y \backslash x=x \backslash y$ iff $X$ is associative.

(49) Let $X$ be a non empty BCI structure with 0 . Then $X$ is an associative BCI-algebra if and only if for all elements $x, y, z$ of $X$ holds $y \backslash x \backslash(z \backslash x)=$ $z \backslash y$ and $x \backslash 0_{X}=x$.

(50) Let $X$ be a non empty BCI structure with 0 . Then $X$ is an associative BCI-algebra if and only if for all elements $x, y, z$ of $X$ holds $x \backslash y \backslash(x \backslash z)=$ $z \backslash y$ and $x^{\mathrm{c}}=x$. 
(51) Let $X$ be a non empty BCI structure with 0 . Then $X$ is an associative BCI-algebra if and only if for all elements $x, y, z$ of $X$ holds $x \backslash y \backslash(x \backslash z)=$ $y \backslash z$ and $x \backslash 0_{X}=x$.

\section{3. $p$-SEMISIMPLE BCI-ALGEBRAS}

One can prove the following propositions:

(52) $X$ is $p$-semisimple iff every element of $X$ is atom.

(53) If $X$ is $p$-semisimple, then BCK-part $X=\left\{0_{X}\right\}$.

(54) $X$ is $p$-semisimple iff for every element $x$ of $X$ holds $\left(x^{\mathrm{c}}\right)^{\mathrm{c}}=x$.

(55) $X$ is $p$-semisimple iff for all $x, y$ holds $y \backslash(y \backslash x)=x$.

(56) $X$ is $p$-semisimple iff for all $x, y, z$ holds $z \backslash y \backslash(z \backslash x)=x \backslash y$.

(57) $X$ is $p$-semisimple iff for all $x, y, z$ holds $x \backslash(z \backslash y)=y \backslash(z \backslash x)$.

(58) $X$ is $p$-semisimple iff for all $x, y, z, u$ holds $(x \backslash u) \backslash(z \backslash y)=y \backslash u \backslash(z \backslash x)$.

(59) $X$ is $p$-semisimple iff for all $x, z$ holds $z^{\mathrm{c}} \backslash x^{\mathrm{c}}=x \backslash z$.

(60) $X$ is $p$-semisimple iff for all $x, z$ holds $\left((x \backslash z)^{\mathrm{c}}\right)^{\mathrm{c}}=x \backslash z$.

(61) $X$ is $p$-semisimple iff for all $x, u, z$ holds $z \backslash(z \backslash(x \backslash u))=x \backslash u$.

(62) $X$ is $p$-semisimple iff for every $x$ such that $x^{\mathrm{c}}=0_{X}$ holds $x=0_{X}$.

(63) $X$ is $p$-semisimple iff for all $x, y$ holds $x \backslash y^{\mathrm{c}}=y \backslash x^{\mathrm{c}}$.

(64) $X$ is $p$-semisimple iff for all $x, y, z, u$ holds $(x \backslash y) \backslash(z \backslash u)=x \backslash z \backslash(y \backslash u)$.

(65) $X$ is $p$-semisimple iff for all $x, y, z$ holds $x \backslash y \backslash(z \backslash y)=x \backslash z$.

(66) $X$ is $p$-semisimple iff for all $x, y, z$ holds $x \backslash(y \backslash z)=(z \backslash y) \backslash x^{\mathrm{c}}$.

(67) $X$ is $p$-semisimple iff for all $x, y, z$ such that $y \backslash x=z \backslash x$ holds $y=z$.

(68) $X$ is $p$-semisimple iff for all $x, y, z$ such that $x \backslash y=x \backslash z$ holds $y=z$.

(69) Let $X$ be a non empty BCI structure with 0 . Then $X$ is a $p$-semisimple BCI-algebra if and only if for all elements $x, y, z$ of $X$ holds $x \backslash y \backslash(x \backslash z)=$ $z \backslash y$ and $x \backslash 0_{X}=x$.

(70) Let $X$ be a non empty BCI structure with 0 . Then $X$ is a $p$-semisimple BCI-algebra if and only if $X$ is I and for all elements $x, y, z$ of $X$ holds $x \backslash(y \backslash z)=z \backslash(y \backslash x)$ and $x \backslash 0_{X}=x$.

\section{Quasi-Associative BCI-Algebras}

Next we state several propositions:

(71) $X$ is quasi-associative iff for every element $x$ of $X$ holds $x^{\mathrm{c}} \leq x$.

(72) $X$ is quasi-associative iff for all elements $x, y$ of $X$ holds $(x \backslash y)^{\mathrm{c}}=(y \backslash x)^{\mathrm{c}}$.

(73) $X$ is quasi-associative iff for all elements $x, y$ of $X$ holds $x^{\mathrm{c}} \backslash y=(x \backslash y)^{\mathrm{c}}$. 
(74) $X$ is quasi-associative iff for all elements $x, y$ of $X$ holds $x \backslash y \backslash(y \backslash x) \in$ BCK-part $X$.

(75) $X$ is quasi-associative iff for all elements $x, y, z$ of $X$ holds $(x \backslash y) \backslash z \leq$ $x \backslash(y \backslash z)$.

\section{Alternative BCI-Algebras}

We now state several propositions:

(76) If $X$ is alternative, then $x^{\mathrm{c}}=x$ and $x \backslash(x \backslash y)=y$ and $x \backslash y \backslash y=x$.

(77) If $X$ is alternative and $x \backslash a=x \backslash b$, then $a=b$.

(78) If $X$ is alternative and $a \backslash x=b \backslash x$, then $a=b$.

(79) If $X$ is alternative and $x \backslash y=0_{X}$, then $x=y$.

(80) If $X$ is alternative and $x \backslash a \backslash b=0_{X}$, then $a=x \backslash b$ and $b=x \backslash a$.

One can check the following observations:

* every BCI-algebra which is alternative is also associative,

* every BCI-algebra which is associative is also alternative, and

* every BCI-algebra which is alternative is also implicative.

The following two propositions are true:

(81) If $X$ is alternative, then $x \backslash(x \backslash y) \backslash(y \backslash x)=x$.

(82) If $X$ is alternative, then $y \backslash(y \backslash(x \backslash(x \backslash y)))=y$.

\section{Implicative, Positive-Implicative, And \\ WEAKLY-Positive-IMPLICATIVE BCI-ALGEBRAS}

Let us observe that every BCI-algebra which is associative is also weaklypositive-implicative and every BCI-algebra which is $p$-semisimple is also weaklypositive-implicative.

We now state two propositions:

(83) Let $X$ be a non empty BCI structure with 0 . Then $X$ is an implicative BCI-algebra if and only if for all elements $x, y, z$ of $X$ holds $x \backslash y \backslash(x \backslash$ $z) \backslash(z \backslash y)=0_{X}$ and $x \backslash 0_{X}=x$ and $(x \backslash(x \backslash y)) \backslash(y \backslash x)=y \backslash(y \backslash x)$.

(84) $X$ is weakly-positive-implicative iff for all elements $x, y$ of $X$ holds $x \backslash y=$ $x \backslash y \backslash y \backslash y^{\mathrm{c}}$.

One can verify that every BCI-algebra which is positive-implicative is also weakly-positive-implicative and every BCI-algebra which is alternative is also weakly-positive-implicative.

One can prove the following two propositions:

(85) Suppose $X$ is a weakly-positive-implicative BCI-algebra. Let $x, y$ be elements of $X$. Then $(x \backslash(x \backslash y)) \backslash(y \backslash x)=y \backslash(y \backslash x) \backslash(y \backslash x) \backslash(x \backslash y)$. 
(86) Let $X$ be a non empty BCI structure with 0 . Then $X$ is a positiveimplicative BCI-algebra if and only if for all elements $x, y, z$ of $X$ holds $x \backslash y \backslash(x \backslash z) \backslash(z \backslash y)=0_{X}$ and $x \backslash 0_{X}=x$ and $x \backslash y=x \backslash y \backslash y \backslash y^{\mathrm{c}}$ and $(x \backslash(x \backslash y)) \backslash(y \backslash x)=y \backslash(y \backslash x) \backslash(y \backslash x) \backslash(x \backslash y)$.

\section{REFERENCES}

[1] Józef Białas. Group and field definitions. Formalized Mathematics, 1(3):433-439, 1990.

[2] Czesław Byliński. Binary operations. Formalized Mathematics, 1(1):175-180, 1990.

[3] Czesław Byliński. Functions and their basic properties. Formalized Mathematics, 1(1):5565, 1990.

[4] Czesław Byliński. Some basic properties of sets. Formalized Mathematics, 1(1):47-53, 1990.

[5] Eugeniusz Kusak, Wojciech Leończuk, and Michał Muzalewski. Abelian groups, fields and vector spaces. Formalized Mathematics, 1(2):335-342, 1990.

[6] Jie Meng and YoungLin Liu. An Introduction to BCI-algebras. Shaanxi Scientific and Technological Press, 2001.

[7] Michał Muzalewski. Midpoint algebras. Formalized Mathematics, 1(3):483-488, 1990.

[8] Michał Muzalewski. Construction of rings and left-, right-, and bi-modules over a ring. Formalized Mathematics, 2(1):3-11, 1991.

[9] Andrzej Trybulec. Subsets of complex numbers. To appear in Formalized Mathematics.

[10] Andrzej Trybulec. Tarski Grothendieck set theory. Formalized Mathematics, 1(1):9-11, 1990.

[11] Wojciech A. Trybulec. Groups. Formalized Mathematics, 1(5):821-827, 1990.

[12] Wojciech A. Trybulec. Vectors in real linear space. Formalized Mathematics, 1(2):291296, 1990.

[13] Zinaida Trybulec. Properties of subsets. Formalized Mathematics, 1(1):67-71, 1990.

[14] Edmund Woronowicz. Relations and their basic properties. Formalized Mathematics, 1(1):73-83, 1990. 